\title{
Hard X-ray properties of magnetic cataclysmic variables
}

\author{
R. Landi ${ }^{* a}$, L. Bassani ${ }^{a}$, L. Amati ${ }^{a}$, A. Bazzano ${ }^{b}$, M. Fiocchi ${ }^{b}$ and A. J. Bird ${ }^{c}$ \\ ${ }^{a}$ INAF - IASF Bologna \\ ${ }^{b}$ INAF - IAPS Rome \\ ${ }^{c}$ University of Southampton \\ E-mail: landiliasfbo.inaf.it, bassaniliasfbo.inaf.it \\ amatidiasfbo.inaf.it angela.bazzanodiaps.inaf.it, \\ mariateresa.fiocchiliasf-roma.inaf.it, A.J.Birdesoton.ac.uk
}

\begin{abstract}
Recent hard X-ray surveys have proven efficient in detecting cataclysmic variables (CVs), in particular magnetic systems (mCVs). This has allowed us to construct a sample of mCVs (mainly Intermediate Polars) selected in hard X-rays, i.e. those observed both by INTEGRAL/IBIS and Swift/BAT. A long-standing issue is the high number of magnetic systems detected among the whole $\mathrm{CV}$ population. Whether this observational evidence is due to selection effects or to different evolutionary time-scales and their connection with the magnetic field of the white dwarf (WD) is still to be explained. The basic idea of this work is to investigate the hard X-ray spectral characteristics of the systems included in the sample and link them to observational features like the orbital and spin periods (Porb, Pspin). In this way, it will be possible to assess their location relative to the period gap, and hence get information about the CVs evolution and accretion geometries on the surface of the white dwarf.
\end{abstract}

An INTEGRAL view of the high-energy sky (the first 10 years)" 9th INTEGRAL Workshop and celebration of the 10th anniversary of the launch,

October 15-19, 2012

Bibliotheque Nationale de France, Paris, France

\footnotetext{
* Speaker.
} 
Sample of hard X-ray selected IPs.

\begin{tabular}{|c|c|c|c|c|c|c|c|}
\hline Source & Type $^{a}$ & BAT & IBIS & Source & Type $^{a}$ & BAT & IBIS \\
\hline IGR J00234+6141 & IP & yes & yes & IGR J15094-6649 & IP & yes & yes \\
\hline V709 Cas & IP & yes & yes & NY Lup & IP & yes & yes \\
\hline 1RXS J005528.0+461143 & IP & yes & no & IGR J16167-4957 & $\mathrm{IP}^{*}$ & yes & yes \\
\hline XY Ari & IP & yes & no & IGR J16500-3307 & IP & yes & yes \\
\hline GK Per & IP & yes & yes & IGR J16547-1916 & $\mathrm{IP}^{*}$ & yes & yes \\
\hline 1RXS J052430.2+424449 & $\mathrm{IP}^{*}$ & yes & no & V2400 Oph & IP & yes & yes \\
\hline TV Col & IP & yes & yes & IGR J17195-4100 & IP & yes & yes \\
\hline TW Pic & $\mathrm{IP}^{*}$ & yes & yes & IGR J17303-0601 & IP & yes & yes \\
\hline TX Col & IP & yes & no & AX J1740.2-2903 & $\mathrm{IP}^{*}$ & no & yes \\
\hline V405 Aur & IP & yes & no & IGR J18173-2509 & $\mathrm{IP}^{*}$ & yes & yes \\
\hline MU Cam & IP & yes & yes & IGR J18308-1232 & IP & no & yes \\
\hline 1RXS J063631.9+353537 & IP & yes & no & AX J1832.3-0840 & $\mathrm{IP}^{*}$ & no & yes \\
\hline BG CMi & IP & yes & no & V1223 Sgr & IP & yes & yes \\
\hline SWIFT J0732.5-1331 & IP & yes & yes & IGR J19267+1325 & $\mathrm{IP}^{*}$ & no & yes \\
\hline PQ Gem & IP & yes & no & IGR J19552+0044 & $\mathrm{IP}^{*}$ & no & yes \\
\hline EL UMa & IP & yes & no & V2306 Cyg & IP & yes & no \\
\hline 1RXS J080114.6-462324 & $\mathrm{IP}^{*}$ & no & yes & 1RXS J211336.1+542226 & $\mathrm{IP}^{*}$ & yes & yes \\
\hline IGR J08390-4833 & IP & yes & yes & V2069 Cyg & IP & yes & yes \\
\hline YY Dra & IP & yes & no & IGR J21335+5105 & IP & yes & yes \\
\hline IGR J12123-5802 & $\mathrm{IP}^{*}$ & no & yes & FO Aqr & IP & yes & yes \\
\hline EX Hya & IP & yes & no & AO Psc & IP & yes & yes \\
\hline
\end{tabular}

${ }^{a} \mathrm{IP}^{*}$ indicates that the IP nature of the objects needs to be still confirmed by measurements.

\section{The sample}

In this preliminary work we have focused our analysis on the sample of IPs detected so far by INTEGRAL/IBIS, Swift/BAT, or both. The number of objects in this sample amounts to 42 and also includes, as shown in the Table, those systems whose characteristics are IP-like, but their class still need to be confirmed.

\section{Spectral analysis results}

As a first step, we analysed the IBIS and BAT spectra in the 20-100 keV energy band independently, adopting a bremsstrahlung model in XSPEC v.12.7.1 (Dorman \& Arnaud 2001). The histograms shown in Figure 1 ( upper-left panel) depict the distribution of the bremsstrahlung temperatures in IBIS (red dashed) and BAT (blue) data set, respectively. The average temperatures are $\langle k T\rangle=23.2 \pm 1.0 \mathrm{keV}$ for the IBIS sample and $\langle k T\rangle=19.5 \pm 1.0 \mathrm{keV}$ for the BAT one. The upper-right panel of Figure 1 shows the correlation between the IBIS and BAT temperatures. It is quite evident from this plot that, within the uncertainties, there is an overall good agreement between the IBIS and BAT temperatures, suggesting that the sources are not affected on average by changes in their spectral shape. In the bottom-left panel of Figure 1 we plot instead the correlation between the IBIS and BAT $20-100 \mathrm{keV}$ fluxes. In this case, despite the correlation turns out to be stringent (correlation coefficient $\sim 0.86$ ), there is a clear indication for the BAT points to be systematically lower than the IBIS ones. This might be due to some systematics between the two instruments, which in AGNs has been already estimated to be around 14\% assuming a power law model (Molina et al. 2012, submitted to MNRAS). 

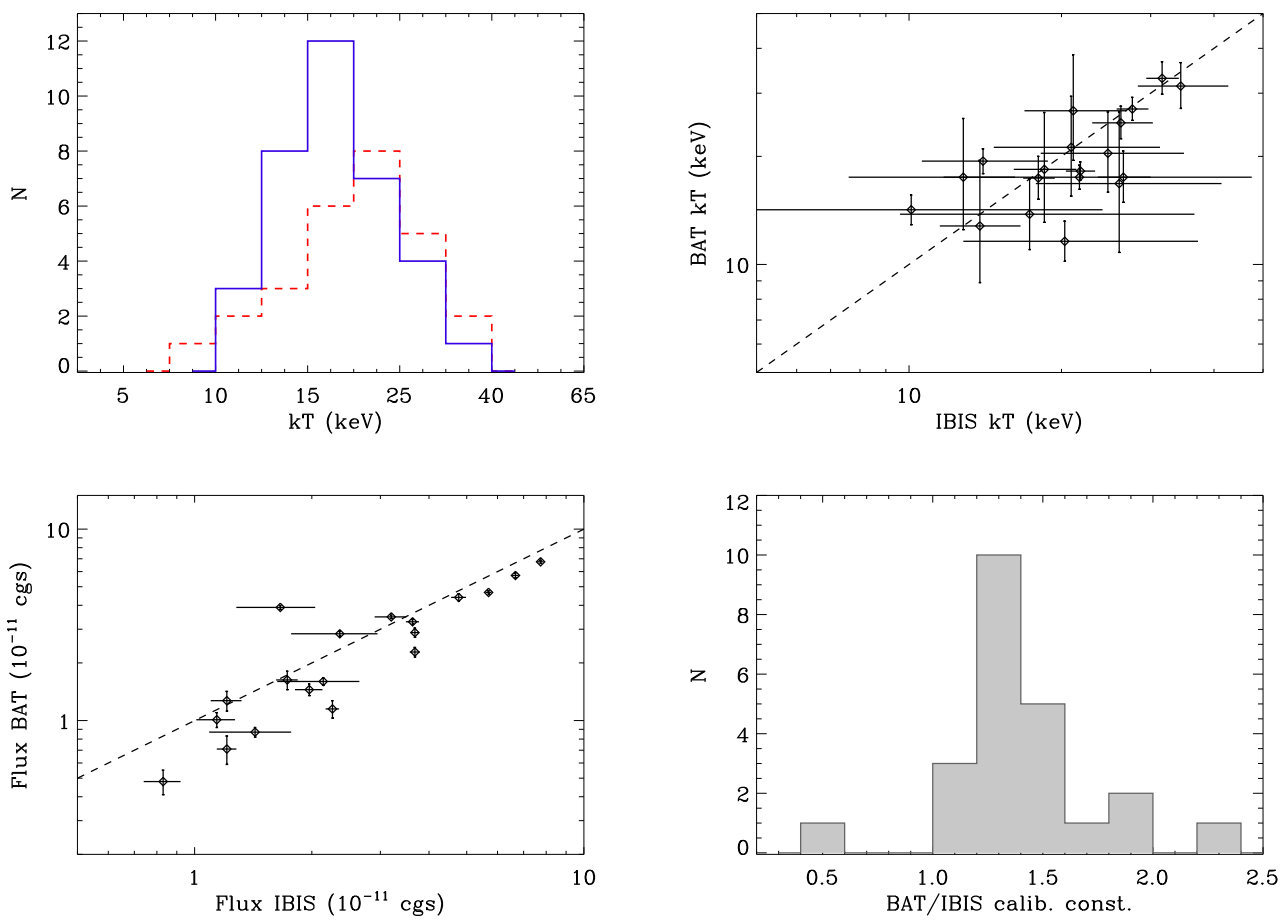

Figure 1: Bremsstrahlung temperature distribution estimated with IBIS (red dashed) and BAT (blue) data set, respectively (upper-left panel). Comparison between the bremsstrahlung temperatures (upper-right panel) and 20-100 keV fluxes (bottom-left panel) derived from individual fits of IBIS and BAT spectra. Histogram of the cross-calibration constant between IBIS and BAT for the selected IPs sample (bottomright panel).

\section{IBIS/BAT simultaneous spectral analysis}

After performing the fit on the individual IBIS and BAT spectra, we combined the two data sets and fit them together. To take into account the presence of flux variability and the possible mis-match between the IBIS and BAT spectra, we have introduced a cross-calibration constant $C$ in the fit. As for the individual data set, the model adopted is a thermal bremsstrahlung. The bottom-right panel of Figure 11 shows the distribution of the cross-calibration constant $C$. It is clear that $C$ is systematically above unity, with an average value of $1.28 \pm 0.02$, similar but not equal to what found in AGNs data (1.14 \pm 0.02 ). The slight difference may be ascribed to different effects, such as the smaller number of objects, different parameters of the model $(k T)$ adopted for the fitting procedure, and possibly a higher source variability in CVs.

\section{Searching for correlations}

A previous study on a smaller sample of IPs (Scaringi et al. 2010) has shown for the first time evidence for a correlation between the hard X-ray spectral hardness and the orbital and spin periods. An attempted explanation was given by Scaringi et al. (2010) in terms of evolution of magnetic systems and accretion geometry onto the WD surface. 

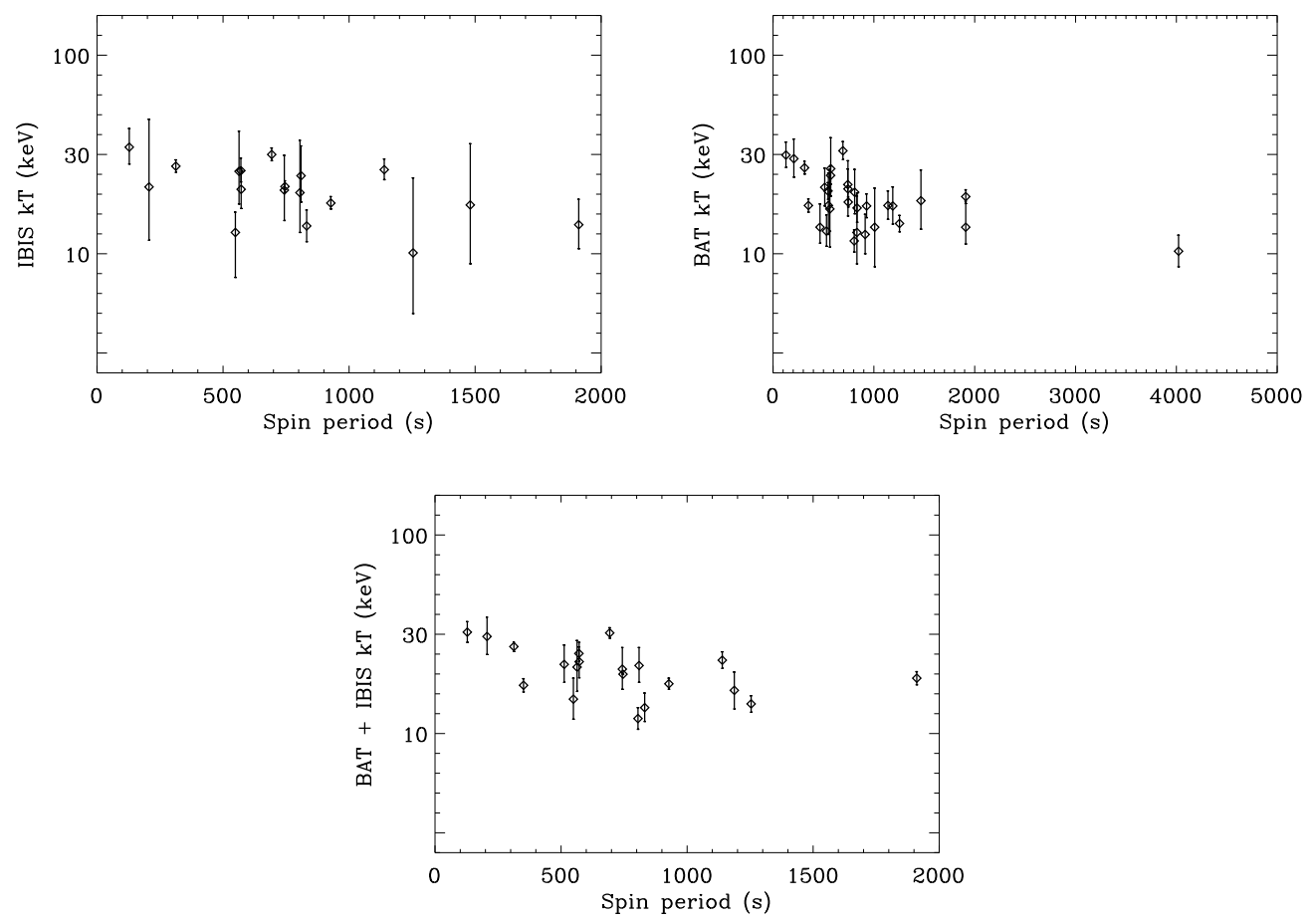

Figure 2: Correlation between IBIS (upper-left panel), BAT (upper-right panel), and joint IBIS/BAT temperatures versus the spin period (bottom-central panel).

In this preliminary work we have extended the sample and searched for correlations between the bremsstrahlung temperature and the orbital parameters, this time using tempertatures rather than axial ratios.

The plots in Figure 2 display the correlation between IBIS (upper-left panel), BAT (upperright panel), and joint IBIS/BAT (bottom-central panel) temperatures versus the spin period. The Spearman rank correlation analysis gives the following probabilities for each data set: $98 \%$ for the IBIS data, $99.4 \%$ for the BAT data, and $99 \%$ for the combined analysis IBIS/BAT. These findings are indicative of a possible anti-correlation which needs to be investigated with further measurements. At this stage, we do not find evidence for a correlation between the bremsstrahlung temperature and the orbital period.

Considering the relevant fraction of mCVs detected at high energies so far, it is likely that they will continue to increase thanks to the contribution that X-ray and optical follow-up observations will give to the identification and classification of still unidentified high energy sources. In particular, more observations will allow us to verify the real existence of the correlation discovered and understand its origin.

\section{References}

[1] Dorman, B. \& Arnaud, K. A., 2001, Astronomical Data Analysis Software and Systems X, ASP Conf. Proc., 238. Eds F. R. Harnden, Jr., F. A. Primini, \& H. E. Payne (San Francisco, CA: ASP), 415

[2] Scaringi, S., Bird, A. J., Norton, A. J., et al, 2012, MNRAS, 401, 2207 